\title{
ARTICLE
}

Received 18 Jun 2010 | Accepted 10 Aug 2010 | Published 7 Sep 2010

DOl:10.1038/ncomms1068

\section{Phase seeding of a terahertz quantum cascade laser} Dimitri Oustinov ${ }^{1}$, Nathan Jukam', Rakchanok Rungsawang', Julien Madéo', Stefano Barbieri², Pascal Filloux²,
Carlo Sirtori², Xavier Marcadet ${ }^{3}$, Jérôme Tignon $\&$ Sukhdeep Dhillon ${ }^{1}$

The amplification of spontaneous emission is used to initiate laser action. As the phase of spontaneous emission is random, the phase of the coherent laser emission (the carrier phase) will also be random each time laser action begins. This prevents phase-resolved detection of the laser field. Here, we demonstrate how the carrier phase can be fixed in a semiconductor laser: a quantum cascade laser ( $Q C L)$. This is performed by injection seeding a $\mathrm{QCL}$ with coherent terahertz pulses, which forces laser action to start on a fixed phase. This permits the emitted laser field to be synchronously sampled with a femtosecond laser beam, and measured in the time domain. We observe the phase-resolved buildup of the laser field, which can give insights into the laser dynamics. In addition, as the electric field oscillations are directly measured in the time domain, QCLs can now be used as sources for time-domain spectroscopy.

\footnotetext{
1 Laboratoire Pierre Aigrain, Ecole Normale Supérieure, CNRS (UMR 8551), Université P. et M. Curie, Université D. Diderot, 75231 Paris Cedex 05, France. 
C ontrolling the carrier-envelope phase of femtosecond (fs) laser pulses by using active feedback techniques ${ }^{1}$ has lead to major advances in frequency metrology ${ }^{2}$ and attosecond science $^{3,4}$. The phase between consecutive electromagnetic pulses is similarly fixed ${ }^{5}$ in time-domain spectroscopy (TDS) ${ }^{6}$. This enables the detection of the electric field in the time domain. As a result, TDS is a powerful technique that allows the dynamics of the complex dielectric response of materials ${ }^{7}$ and coherent processes ${ }^{8}$ to be investigated as a function of time. In the terahertz $(\mathrm{THz})$ frequency regime, TDS is a widely used technique for THz imaging ${ }^{9}$, biological investigations ${ }^{10}$, non-destructive testing ${ }^{11}$ and chemical recognition ${ }^{12}$. THz TDS is implemented by generating $\mathrm{THz}$ pulses with a fs laser beam, and synchronously sampling the resulting $\mathrm{THz}$ pulses with the same fs laser beam, using techniques such as free-space electro-optic sampling ${ }^{13}$. Time-domain detection of the electric field is made possible, since the phase between successive $\mathrm{THz}$ pulses is fixed, even if the carrier-envelope phase of the fs laser pulses is not fixed ${ }^{5}$. This allows multiple-shot averaging of the sampled electric field value. By varying the time delay between the sampling beam and the $\mathrm{THz}$ pulse, the field can be characterized as a function of time.

However, time-domain detection of free-running sources, such as quantum cascade lasers (QCL) ${ }^{14,15}$, cannot be performed with an fs sampling beam. Even with an infinite coherence time, the QCL frequency would need to be an integer multiple of the fs sampling rate (the repetition rate of the fs laser). Otherwise, the carrier phase would vary for each fs sampling pulse, and the resulting multipleshot average of the sampled electric field value would be zero. Thus, the field of a free-running QCL cannot be measured as a function of time and cannot be used as a source for TDS. This is unfortunate, as many emerging $\mathrm{THz}$ applications ${ }^{16,17}$ take advantage of the small size and high output power ${ }^{18}$ of this semiconductor laser.

In this article, we show how the carrier phase of the QCL laser emission can be set by coherently injection seeding ${ }^{19}$ the QCL with $\mathrm{THz}$ pulses having a fixed phase. Here, the seeded field replaces the spontaneous emission of the QCL and sets the carrier phase by triggering the laser emission. Consequently, this enables the electric field of the laser emission to be measured as a function of time over several nanoseconds, from initiation of lasing to the steady-state lasing regime. In contrast to other techniques ${ }^{2,17,19}$ no active feedback or laser stabilization mechanism is necessary for phase-controlled emission.

\section{Results}

To initiate lasing, coherent $\mathrm{THz}$ pulses are injected into the QCL when the gain is below threshold, as depicted in Figure 1a. A short time later, the QCL gain is increased above threshold by applying a voltage pulse (radiofrequency (RF) pulse) to the QCL. As no laser field is initially present in the QCL cavity, the gain is unclamped and large amplification can occur ${ }^{20}$. Both the $\mathrm{THz}$ pulse and the spontaneous emission in the cavity will be amplified until the intracavity field is large enough to saturate the gain. This clamps the gain to the total losses and results in steady-state lasing. If the field of the injected THz pulses is greater than the spontaneous emission, lasing will be initiated by the $\mathrm{THz}$ pulses. The $\mathrm{THz}$ pulses will then seed the lasing, as shown in Figure 1a. The carrier phase of the QCL is therefore set by the phase of the coherent $\mathrm{THz}$ pulses. This enables the field of the QCL to be measured in the time domain, as shown in Figure 1b. If no injected THz pulse is present, for each RF pulse, the phase of the spontaneous emission (which is random) determines the carrier phase of the QCL, and time-domain detection of the laser emission is not possible, as shown in Figure 1c. It should be noted that, even if the carrier phase of the fs laser pulses varies from pulse to pulse, the phase of the generated $\mathrm{THz}$ pulses does not vary (Fig. 1d). This is because the THz pulses are created in response to the rectified component of the instantaneous fs laser intensity, which does not depend on the carrier phase of the fs laser (Fig. 1e). a

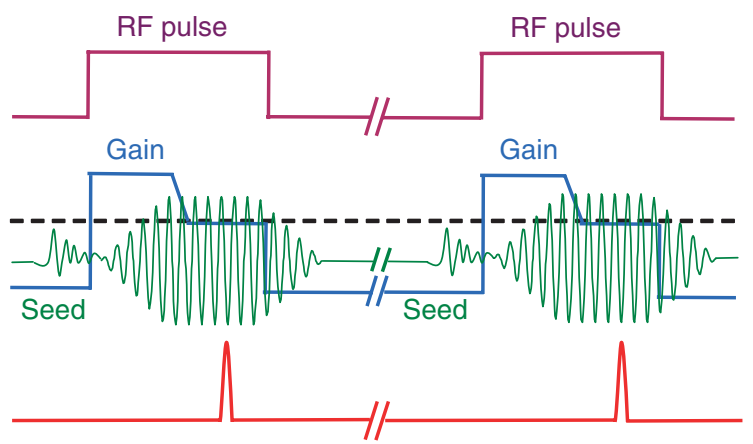

fs Sampling pulse

fs Sampling pulse
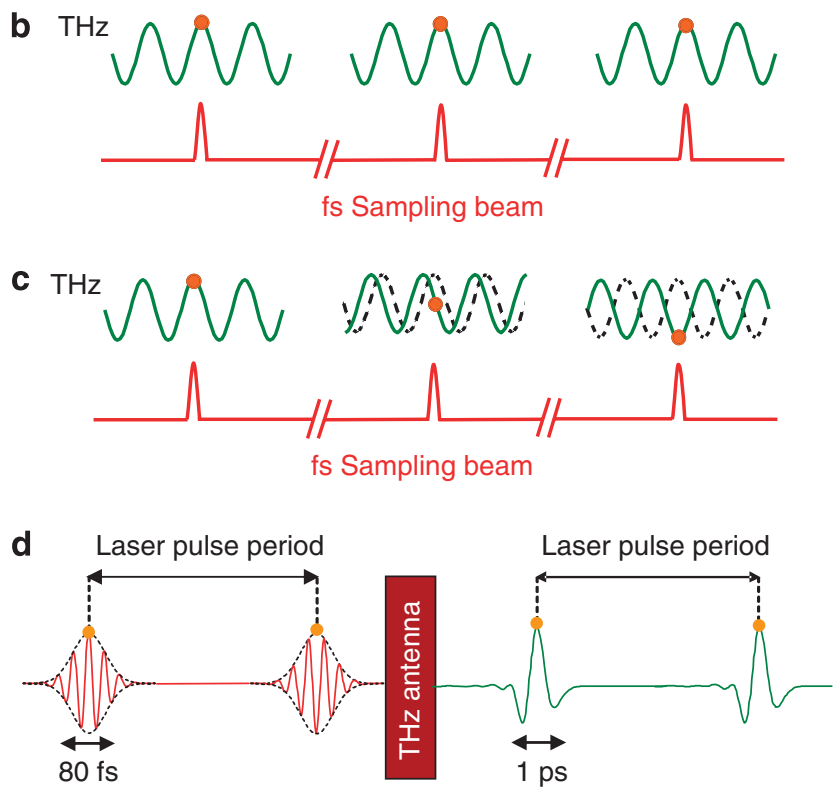

e

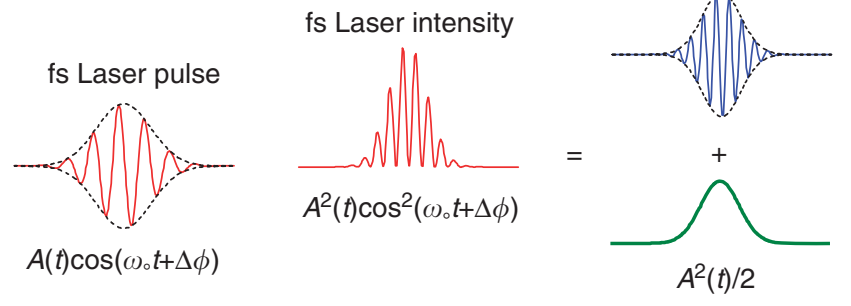

Figure 1 | Coherent injection seeding of a QCL. (a) A THz pulse injected into the $\mathrm{QCL}$ before the gain is turned on initiates lasing. The carrier phase of the QCL emission is then set by the injected THz pulse. (The dashed line represents the value of the gain at laser threshold.) (b) The carrier phase of the emission from a QCL is synchronized with respect to a femtosecond (fs) sampling beam. (c) The carrier phase of the emission from a freerunning QCL is not synchronized with respect to a femtosecond sampling beam. The femtosecond sampling beam will measure an average electric field of zero. (d) The phase of the THz pulses is fixed from pulse to pulse even if the carrier phase of the femtosecond laser pulses varies. (e) $\mathrm{THz}$ pulses are generated in response to the envelope function (green) of the intensity of the femtosecond laser pulse, which does not depend on the carrier phase. Only the second harmonic response (blue) of the instantaneous intensity depends on the carrier phase $\Delta \phi$. However, it has no role in the generation of terahertz radiation. 

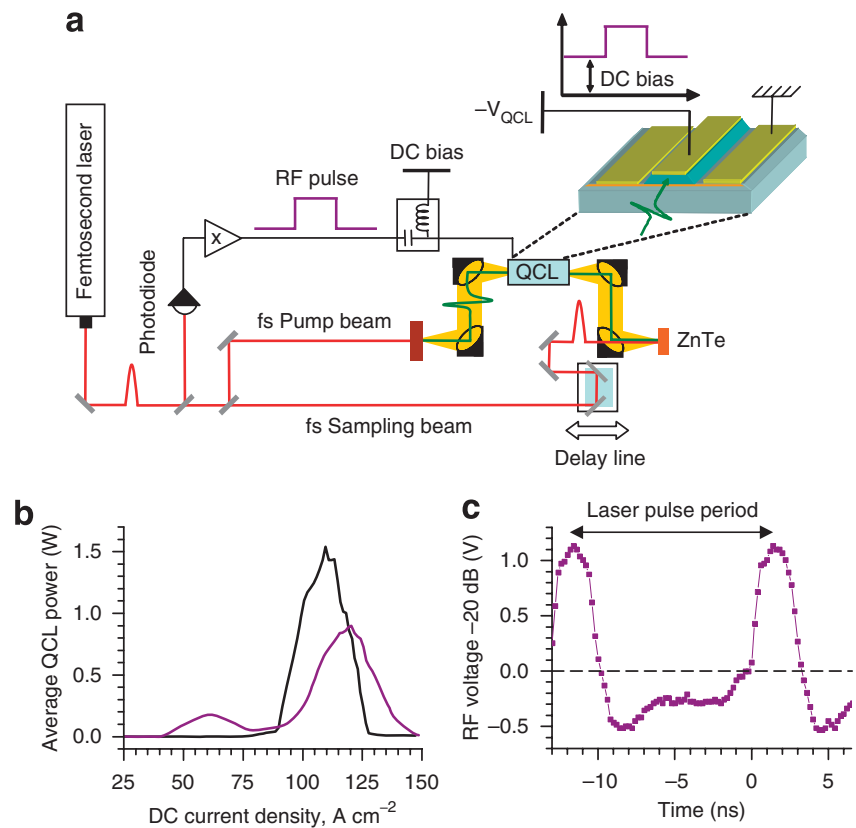

Figure 2 | Experimental setup. (a) Schematic of the experimental setup. RF voltage pulses are generated from a fast photodiode illuminated by the femtosecond ( $\mathrm{fs}$ ) laser beam (with a centre wavelength of $820 \mathrm{~nm}$ ). The RF pulses are amplified by a power RF amplifier. A bias tee adds a DC offset to the RF pulses. THz pulses are generated by illuminating a biased interdigitiated antenna with a femtosecond pump beam. The $\mathrm{THz}$ pulses are coupled into a facet of the quantum cascade laser $(\mathrm{QCL})$ with parabolic mirrors. The QCL output field from the other facet is measured using electro-optic sampling in a ZnTe crystal with a femtosecond sampling beam. (b) Time-averaged light-current density ( $L-J$ ) curve of the QCL with RF OFF (black) and RF ON (purple). The QCL output power is measured with a slow pyroelectric detector that averages out any power variations caused by the RF pulses. (c) Instantaneous voltage of RF pulses, measured with a $1 \mathrm{GHz}$ oscilloscope and $20 \mathrm{~dB}$ attenuation. The two peaks correspond to two consecutive laser pulses. The negative voltages between RF pulses arise because the RF pulses are alternate current (AC) coupled and the time-averaged RF voltage must therefore be zero.

Experimental setup. The RF pulses are created by illuminating a fast photodiode with a portion of the fs laser beam. This ensures that the RF pulses are synchronized with the $76 \mathrm{MHz}$ pulse train of the fs laser. The RF pulses are amplified, and a quasi-direct current (DC) offset is then added to the amplified RF pulses with a bias tee. The length of the coaxial cable (which carries the RF pulses) was precisely adjusted so that the edge of the RF pulses arrived at the same time as the injected $\mathrm{THz}$ pulses. Figure $2 \mathrm{a}$ shows a schematic of the experimental setup. THz pulses are generated by illuminating an interdigitated photoconductive antenna ${ }^{21}$ with the fs laser beam. The THz pulses are coupled into a facet of a $2 \mathrm{~mm}$-long bound-tocontinuum QCL ${ }^{22}$ operating at $\sim 2.1 \mathrm{THz}$. At the opposite facet, the output from the QCL is collected and focused onto a ZnTe crystal along with the fs sampling beam. The $\mathrm{THz}$ field inside the $\mathrm{ZnTe}$ crystal is then measured using electro-optic sampling ${ }^{13}$.

The time-average light-current density (L-J) curves are shown with (purple) and without (black) RF pulses applied to the QCL in Figure $2 \mathrm{~b}$. With RF pulses applied to the QCL, a local maximum in the time-average L-J curves appears at low current densities. This corresponds to the RF pulses turning on the QCL, with the DC bias below threshold. Figure $2 c$ shows the instantaneous voltage of a typical RF pulse as a function of time. As the RF pulses are amplified by an alternate current (AC) amplifier with a bandwidth of $30 \mathrm{MHz}$ to $1.2 \mathrm{GHz}$, the time-averaged voltage must be zero. This can be seen in Figure 2c, in which the RF voltage is negative between RF pulses. The negative RF voltage between pulses causes the global maximum of the time-average L-J curve to shift to higher DC current densities in Figure $2 b$ when the RF pulses are on.

When a THz pulse is coupled into the input facet of the QCL, it makes a single pass through the QCL. At the output facet, part of the $\mathrm{THz}$ pulse is reflected back into the cavity of the QCL, and the other part is transmitted out of the QCL. A portion of the reflected THz pulse will make another round-trip through the cavity and return to the output facet. This process is repeated many times, and the output from the QCL consists of multiple $\mathrm{THz}$ pulses separated by the round-trip time of the laser cavity.

QCL output with the RF pulses OFF. Figure 3a (blue curve) shows the transmitted $\mathrm{THz}$ pulses with the RF pulses OFF and the QCL biased above the lasing threshold. The THz pulses become elongated and their amplitude becomes smaller as they undergo more and more passes through the QCL. This is similar to previous studies in which $\mathrm{THz}$ probe pulses are used to measure the spectral gain of $\mathrm{QCLs}^{23-26}$. In these studies, TDS can only measure the amplification of the injected THz probe pulses. The emission of the QCL cannot be detected, as the carrier phase of the QCL is not locked to the fs sampling rate (the repetition rate of the fs laser). This is clearly evident in Figure 3a; no sinusoidal steady-state laser field can be measured even though the QCL is biased above threshold and lasing.

QCL output with the RF pulses ON. In strong contrast to the data in Figure 3a, when the DC bias of the QCL is below threshold, but the RF pulses are ON, the amplitude of successive $\mathrm{THz}$ pulses in Figure $3 \mathrm{~b}$ (green curve) significantly increases. The $\mathrm{THz}$ pulses increase in amplitude for $\sim 400$ ps (from 200 to $600 \mathrm{ps}$ ), which is on the order of the rise time of the RF pulses. For much longer time periods, successive $\mathrm{THz}$ pulses merge together, and the field becomes quasi-monochromatic, as shown in Figure 3c. At these time scales, the THz field in the QCL cavity has saturated the gain (clamped it to the total losses), and the injected $\mathrm{THz}$ seed pulse has become the laser field of the QCL. The field amplitude emitted by the QCL (in the absence of injected THz pulses) is calculated from power measurements to be $8.1 \mathrm{~V} \mathrm{~cm}^{-1}$. This is in good agreement with the measured amplitude $\left(6.4 \mathrm{~V} \mathrm{~cm}^{-1}\right)$ in Figure $3 \mathrm{c}$, considering that the frequency response of the ZnTe crystal is not taken into account. A small secondary $\mathrm{THz}$ pulse, caused by reflections in the substrate of the THz-generating antenna, can be observed at $\sim 35$ ps in Figure 3a. The secondary THz pulse is also amplified by the unclamped gain and alters the shape of the field envelope as the field in the QCL builds up. Interference from the secondary seed pulse also contributes to the ripples of the field envelope at much longer time periods ( $>800 \mathrm{ps}$ ) in Figure $3 \mathrm{~b}$.

In Figure 3d, the field is shown for the entire scan of 2,200 ps. After the initial rise of the $\mathrm{THz}$ pulses, a slight decrease of the peak field is observed after 600 ps. This could be due to a small overshoot of the $\mathrm{THz}$ field with respect to the steady-state lasing value, or to a voltage deviation of the RF pulse from an ideal rectangular pulse shape (as shown in Fig. 2c). After a slight decrease of the peak amplitude (that takes place after $600 \mathrm{ps}$ ), the peak amplitude is approximately flat until $\sim 2,000 \mathrm{ps}$, at which time the RF pulse begins to turn OFF. In Figure 3e, the spectral amplitude of the field in Figure $3 \mathrm{~d}$ is compared with the spectral amplitude of an input $\mathrm{THz}$ pulse produced by the THz-generating antenna, with the QCL removed from the setup. The spectral amplitude of the QCL field (measured by electro-optic sampling) is two orders of magnitude larger than that of the antenna at $2.13 \mathrm{THz}$. In the inset of Figure $3 e$, it can be seen that the measured QCL field consists of a main line at $2.13 \mathrm{THz}$ surrounded by several smaller lines with a spacing of $\sim 20 \mathrm{GHz}$, which corresponds to the round-trip time ( $\sim 50 \mathrm{ps})$ of the laser cavity. 

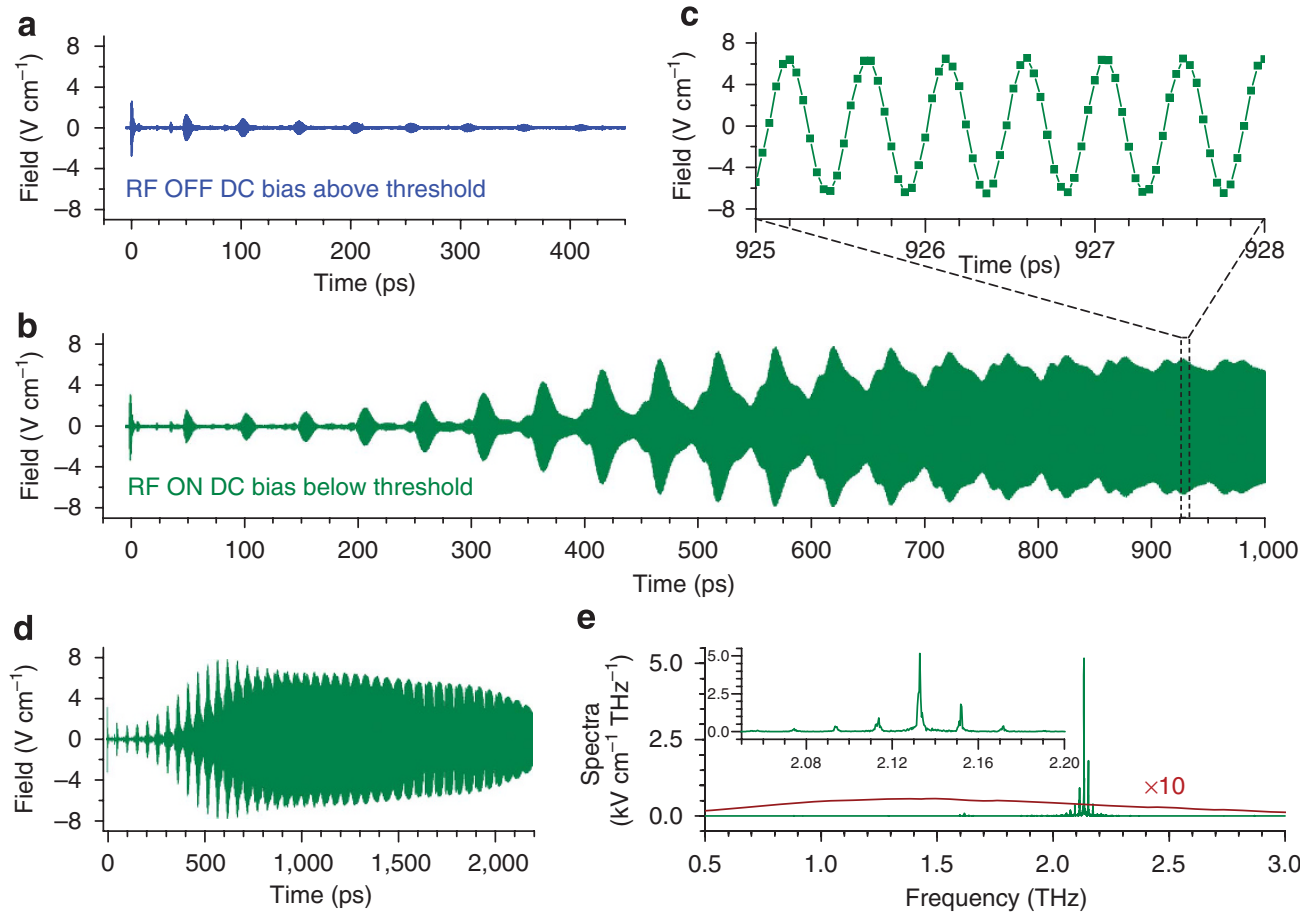

Figure 3 | Measured fields emitted from the QCL with and without RF pulses. (a) THz pulses transmitted through the QCL, with the RF pulses OFF and the DC bias $\left(93 \mathrm{~A} \mathrm{~cm}^{-2}\right.$ ) above threshold. (b) THz pulses transmitted through the QCL, with the RF pulses ON and the DC bias (60 A cm $\left.{ }^{-2}\right)$ below threshold. (c) Closeup of electro-optically measured field in part b from 925 to $928 \mathrm{ps}$. (d) The electro-optically measured QCL field for the entire scan of 2,200 ps. (e) Spectral amplitude of the electro-optically measured QCL field (green curve) and the antenna output with the QCL removed from the setup (dark red curve). The antenna spectrum is multiplied by a factor of 10 for visual clarity. The inset shows a closeup of the spectral amplitude of the electrooptically measured QCL field.

Saturation measurements. To further confirm that injection seeding is taking place, saturation measurements are performed on the QCL by varying the amplitude of the input THz pulses. To change the amplitude of the $\mathrm{THz}$ pulses, we varied the voltage applied to the $\mathrm{THz}$-generating antenna. The amplitude of the $\mathrm{THz}$ pulses was confirmed to be proportional to the voltage applied to the antenna (see Supplementary Figure S1). For the data in Figure 3, the antenna voltage was $4 \mathrm{~V}$.

Figure 4a shows output fields of the QCL for different amplitudes of the input $\mathrm{THz}$ pulses. For each field in Figure $4 \mathrm{a}$, the antenna voltage, and hence the input $\mathrm{THz}$ amplitude, varies by a factor of 4. It is particularly striking that for time periods $>500$ ps (after the intracavity field buildup time), the output amplitude hardly changes for antenna voltages of 0.25 and $1 \mathrm{~V}$, even though the input $\mathrm{THz}$ pulse amplitude differs by a factor of 4 . The root mean square and peak-to-peak (p-p) amplitude of the fields from 500 to 640 ps are plotted in Figure $4 \mathrm{~b}$ as a function of the antenna voltage. The kink in Figure $4 \mathrm{~b}$ at $0.25 \mathrm{~V}$ shows a clear saturation of the output field. This implies that the QCL gain is clamped (that is, saturated) by the amplified $\mathrm{THz}$ input pulses, and the $\mathrm{THz}$ input pulses initiate (that is, seed) laser action for antenna voltages $>0.25 \mathrm{~V}$. For antenna voltages $<0.25 \mathrm{~V}$, the measured QCL output field depends on the input $\mathrm{THz}$ pulse amplitude. This implies that the QCL gain is then clamped by the intracavity amplified spontaneous emission, rather than by the injected THz pulses. As the amplified spontaneous emission has a carrier phase that varies randomly for each fs sampling pulse, its field cannot be detected with the fs sampling beam.

Seed amplification. A laser can be considered as the ultimate amplifier: when initially turned on, it takes a very small signal (the spontaneous emission), and amplifies it to the steady-state laser value. Injection seeding can be simply thought of as amplification of an input seed to the value of the laser field. We calculate the amplification factor using the QCL field from one round-trip time as the output, and the THz field from the antenna (with the QCL removed from the setup) as the input. The power amplification factor is shown in Figure 5a with and without taking account of the large coupling losses into the QCL facet. The largest amplification factor occurs for $\mathrm{THz}$ input pulses generated with antenna voltages $<0.25 \mathrm{~V}$. These $\mathrm{THz}$ pulses are in the linear amplification regime of Figure $5 \mathrm{a}$ and do not saturate the QCL gain. As a consequence, the amplification factor is relatively flat in Figure $5 \mathrm{a}$ for antenna voltages $<0.25 \mathrm{~V}$. In contrast for $\mathrm{THz}$ input pulses with antenna voltages $>0.25 \mathrm{~V}$, the amplification factor decreases as the $\mathrm{THz}$ pulse amplitude increases, as these THz pulses saturate the QCL output. The QCL output spectra from one round-trip time interval (557-608 ps) and the $\mathrm{THz}$ input pulse spectra are shown in Figure $5 \mathrm{~b}$ and $\mathrm{c}$ for antenna voltages of 0.25 and $0.03 \mathrm{~V}$. Unlike Figure $3 e$, the QCL output spectra in Figure $5 b$ and $c$ both consist of a single peak. The spectral lines in Figure $3 \mathrm{e}$ are the result of multiple reflections from the laser cavity facets and are only present for time durations greater than the round-trip time.

\section{Discussion}

In the measurements presented above, the QCL is seeded by an external $\mathrm{THz}$ pulse from a photoconductive antenna. The latter can be easily eliminated by directly generating the THz pulse on the QCL by exciting the facet with a fs laser puls $\mathrm{e}^{27}$. As well as simplifying the TDS setup, this would avoid the large coupling losses associated with injecting free-space $\mathrm{THz}$ radiation into QCL waveguides, as the $\mathrm{THz}$ pulse would be automatically coupled into the QCL cavity. Furthermore, the technique of coherent injection seeding is not limited to the far infrared. It should also be possible to phase-seed mid-infrared QCLs and detect their phase-resolved output in the time domain. 

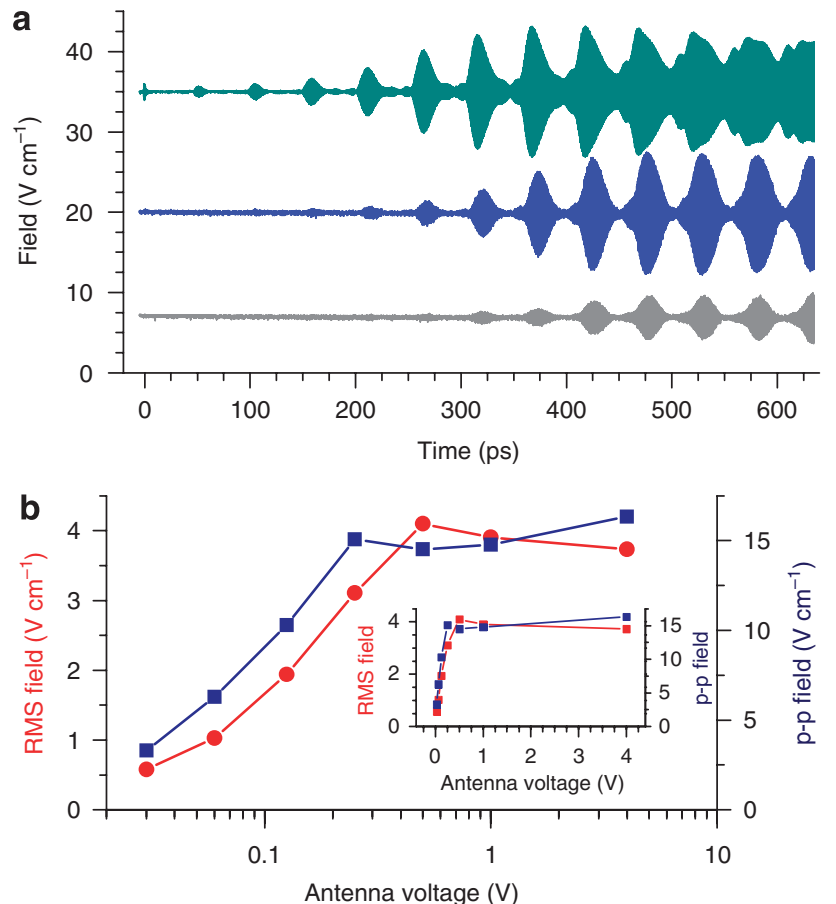

Figure 4 | Seed pulse saturation measurements. (a) Electro-optically measured fields of the QCL output for various input $\mathrm{THz}$ pulse amplitudes. The $\mathrm{THz}$ pulse amplitude is varied by changing the antenna voltage. ( $T$ he $\mathrm{THz}$ pulse amplitude is proportional to the antenna voltage.) Output fields are shown for input $\mathrm{THz}$ pulses generated with an antenna bias of $1 \mathrm{~V}$ (green curve), $0.25 \mathrm{~V}$ (blue curve) and $0.06 \mathrm{~V}$ (grey curve). The QCL DC bias $\left(66 \mathrm{~A} \mathrm{~cm}^{-2}\right.$ ) is below threshold. (b) The peak-to-peak ( $\left.p-p\right)$ amplitude and root mean square (RMS) of the THz fields in part a for time periods $>500$ ps versus the antenna voltage (logarithmic scale). Inset-part $\mathbf{b}$, on a linear voltage scale.

Indeed, investigations of the gain of mid-infrared QCLs using TDS have already begun ${ }^{26,28}$. Besides using the QCL as an active device, the phase-resolved dynamics of the QCL field can also be explored. This could enable the investigation of mode-locked behaviour in QCLs. ${ }^{29,30}$ The dynamics of the buildup of the laser field can also be investigated from the initiation of lasing to the steady state.

It is interesting to consider the results in the context of the frequency domain. For very long time scales, the output of the injection-seeded QCL consists of a train of THz pulses (with nanosecond durations) emitted at the fs laser repetition rate ( $\sim 13 \mathrm{~ns})$. The spectrum of this QCL pulse train should mathematically consist of several spectral lines $\left(f_{\mathrm{QCL}}\right)$, such that $f_{\mathrm{QCL}}=m f_{\text {rep }}+f_{\mathrm{o}}$, where $\mathrm{m}$ is an integer, $f_{\text {rep }}$ is the fs laser repetition frequency and $f_{\mathrm{o}}$ is an offset frequency ${ }^{1}$. If the carrier phase is constant and does not vary between pulses, the frequency offset $\left(f_{\mathrm{o}}\right)$ will be exactly equal to zero. ${ }^{1}$ This enables synchronized fs sampling of the QCL emission, and timeresolved detection of the QCL field. In contrast, for non-synchronized fs sampling ${ }^{31}$ and other phase-locked QCLs ${ }^{32}, f_{\mathrm{o}}$ is not zero, which inhibits time-resolved detection of the field.

In conclusion, the carrier phase of a QCL can be set by coherent injection seeding without any active feedback or laser stabilization techniques. This enables the phase-resolved laser emission to be measured in the time domain and the QCL to be used as a powerful source for TDS.

\section{Methods}

Description of the QCL. The QCL device was a $2 \mathrm{~mm}$-long and $250 \mu \mathrm{m}$-wide QCL that lased at $\sim 2.1 \mathrm{THz}$. A plasmon waveguide was used for mode confinement.
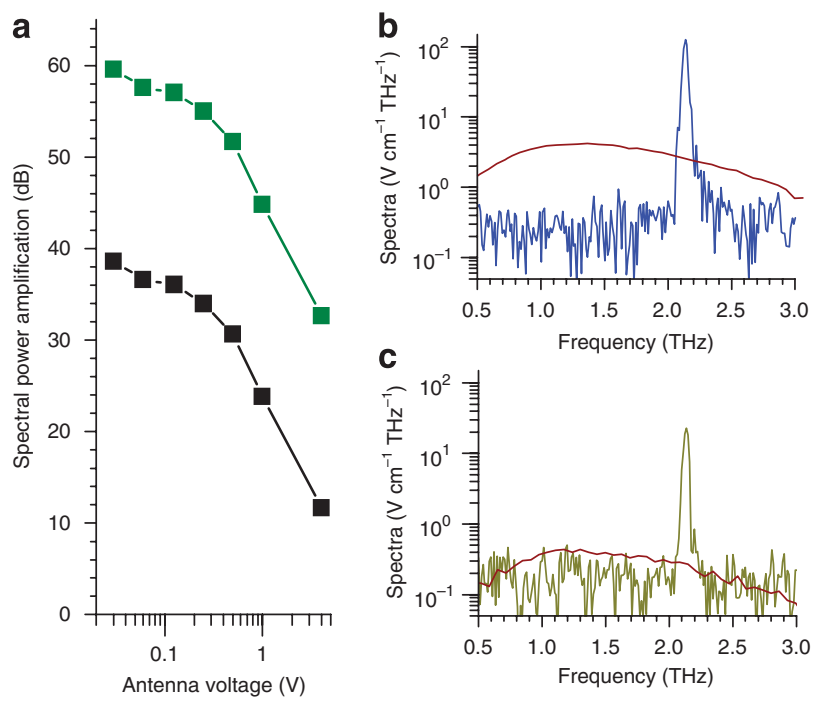

Figure 5 | Amplification of the seed pulse. (a) Power spectral amplification factors for the fields in Figure 4 (from 557 to 608 ps; one round-trip time interval) versus antenna voltage. Green-including input coupling losses. Black-excluding input coupling losses. The THz pulse amplitude is proportional to the antenna voltage. (b) Antenna voltage $0.25 \mathrm{~V}$. Blue curve-spectra of the QCL output from 557 to 608 ps. Dark red curve-spectra of the antenna output with the QCL removed from the setup. (c) Antenna voltage 0.03 V. Dark yellow curve-spectra of the QCL output from 557 to 608 ps. Dark red curve-spectra of the antenna output with the QCL removed from the setup.

The active region of the device consisted of alternatively spaced layers of GaAs and $\mathbf{A l}_{10} \mathbf{G a}_{90} \mathbf{A s}$, with thicknesses of 5/126/44/120/32/124/30/132/24/144/10/118/10/14 $4 \mathrm{~nm}$. The device was bounded to a $\mathrm{Cu}$ heat sink bar with indium and attached to a cold finger of a helium flow cryostat that was cooled to $4.5 \mathrm{~K}$.

THz pulse generation and detection. THz pulses were generated by illuminating an interdigitated photoconductive antenna on GaAs with an fs laser $(\sim 1 \mathrm{~W}$ average power, $\sim 80 \mathrm{fs}$ pulse duration, $76 \mathrm{MHz}$ ). The antenna had a finger spacing of $1.5 \mu \mathrm{m}$ and a period of $6 \mu \mathrm{m}$. The macroscopic active area of the antenna was a $500 \mu \mathrm{m}$ circle. The $500 \mu \mathrm{m}$-thick GaAs substrate was attached to a $1 \mathrm{~mm}$ high-resistivity $\mathrm{Si}$ wafer to extend the echo time between multiple reflections in the substrate. The generated $\mathrm{THz}$ pulses were collected by a $\# 2$ parabolic mirror and focused onto a QCL facet with a $\# 1.67$ parabolic mirror. The windows of the cryostat were thin quartz windows, unlike the tsurupica windows used in previous studies. After exiting the QCL through the opposite facet, the THz pulses were collimated with a $f \# 2$ parabolic mirror and refocused with a f\#1 parabolic mirror onto a $200 \mu \mathrm{m}$-thick $\langle 110\rangle$ ZnTe crystal. The ZnTe crystal was mounted on a 2 mm-thick $\langle 100\rangle$ ZnTe crystal (The $\langle 100\rangle$ crystal has a null electro-optic effect in this geometry.) This extended the time between echoes in the ZnTe. The THz electric field in the $200 \mu \mathrm{m}$ ZnTe crystal was then measured with an fs sampling beam using electro-optic sampling.

Generation and amplification of RF voltage pulses locked to the fs laser repetition rate. $\mathrm{RF}$ voltage pulses were created by illuminating a fast $\mathrm{Si}$ photodiode with a portion of the fs laser beam. The RF pulses were sent into a preamplifier that was used as a limiting amplifier to convert the RF pulses to square-like pulses with a relatively flat top. A $50-\Omega$ switch enabled the RF pulse train to be gated at $\mathrm{kHz}$ frequencies. A variable voltage-controlled attenuator was used to vary the amplitude of the RF pulses, which were then sent into an RF power amplifier $(30-1,200 \mathrm{MHz})$. Fixed coaxial attenuators were used throughout the RF setup to control the RF power level. The amplified RF pulses were then combined with a $\mathrm{DC}$ bias (modulated at $10 \mathrm{kHz}$ ) in a bias tee. The DC bias was modulated at $10 \mathrm{kHz}$ to enable detection with a lock-in amplifier. The same $10 \mathrm{kHz}$ modulation was also applied to the RF pulses with the $50-\Omega$ switch. The combined RF pulses and the quasi-DC offset were applied to the QCL sample. The leads connecting the sample to the coaxial cable were made as short as possible to avoid RF reflections. The RF input pulses and reflected RF output pulses could be monitored by using a two port 20 - $\mathrm{dB}$ directional coupler $(40-1,000 \mathrm{MHz})$. The RF pulses split off by the directional coupler were measured with a $1 \mathrm{GHz}$ oscilloscope across a $50 \Omega$ load.

Adjusting the arrival time of the RF electrical pulses. To ensure that the arrival of the RF pulses coincided with the arrival of the THz pulses into the QCL cavity, the length of the coaxial cable in the RF setup was adjusted. Fine-length adjustment 
could be made by adding and subtracting several Sub miniature version A (SMA) male-female adapters. Because of the amount of RF components and the geometry of the experimental setup, the path length for the RF pulses was much longer than the path length for the fs and THz pulses. Because of this, a single fs laser pulse could not be used to trigger the same THz pulse and RF pulse. Instead the THz pulses were triggered two laser pulses ( $26 \mathrm{~ns})$ after the RF electrical pulses were triggered.

Modulation and data-taking scheme for lock-in detection. The antenna was modulated at $20 \mathrm{kHz}$ with a $50 \%$ duty cycle and the QCL and $50 \Omega$ switch bias pulses were modulated at $10 \mathrm{kHz}$ with a $25 \%$ duty cycle. The individual QCL bias pulses and the antenna bias pulses had the same time duration, and were synchronized in time. There were twice as many antenna bias pulses as QCL bias pulses, and for every other antenna bias pulse, the QCL bias pulse was alternatively ON and then OFF. The $10 \mathrm{kHz}$ modulation was used as the reference for the lock-in amplifier. Because of the synchronized bias pulse scheme, the lock-in amplifier detected the difference between the THz field with the QCL and RF ON, and the THz field with the QCL and RF OFF $\left(E(t)_{\mathrm{QCL}, \mathrm{RF}} \mathrm{ON}-E(t)_{\mathrm{QCL}, \mathrm{RF}} \mathrm{OFF}\right)$. However, this distinction is only important for a single pass through the QCL. For multiple passes, the loss within the QCL is so great that $E(t)_{\mathrm{QCLRF}}$ ofF is essentially zero, and the measured field is for all practical purposes $E(t)_{\mathrm{QCL}, \mathrm{RF}}$ ON.

Steady-state value determination for the field emitted by the QCL. We can determine whether the QCL field (measured by electro-optic detection in Figure 3c) is comparable with the field emitted by the QCL in the steady-state regime (with no THz pulses injected into the laser). To do this, we measure the c.w. power of the THz pulse generated by the antenna $\left(P_{\mathrm{Ant}}\right)$ with the QCL removed from the setup, and the c.w. power of the QCL emission $\left(P_{\mathrm{QCL}}\right)$ (with no seeding THz pulses) under identical conditions with a pyroelectric detector. If we assume that the spot size of the QCL emission and the THz pulses are identical in the ZnTe crystal, then

$$
\sqrt{\frac{P_{\mathrm{QCL}}}{P_{\mathrm{Ant}}}} \equiv \frac{\sqrt{\left\langle E_{\mathrm{QCL}}^{2}(t)\right\rangle}}{\sqrt{\frac{1}{T} \int_{0}^{T} E_{\text {Ant }}^{2}(t) \mathrm{d} t}}
$$

where $E_{m}(t)$ is the field of the THz pulse from the antenna (with the QCL removed), $E_{\mathrm{QCL}}(t)$ is the field of the c.w. QCL emission and $T$ is the period of the fs laser pulse ( $\sim 13 \mathrm{~ns})$. By measuring $E_{\mathrm{Ant}}(t)$ using electro-optic sampling, the root mean square of the THz pulse can be determined, and the amplitude of the c.w. QCL emission can be calculated. Note that, besides implicitly assuming identical spot sizes in the ZnTe crystal, equation (1) also neglects the frequency response of the ZnTe and pryoelectric detectors. We find an amplitude of $8.1 \mathrm{~V} \mathrm{~cm}^{-1}$ for the steady-state QCL field, which is in good agreement with the time-resolved electrooptically measured field of $6.4 \mathrm{~V} \mathrm{~cm}^{-1}$ in Figure $3 \mathrm{c}$.

\section{References}

1. Jones, D. J. et al. Carrier-envelope phase control of femtosecond mode-locked lasers and direct optical frequency synthesis. Science 288, 635-639 (2000).

2. Holzwarth, R., Reichert, J., Udem, Th. \& Hansch, T. W. A new type of frequency chain and its application to optical frequency metrology. Laser Phys. 11, 1100-1109 (2001).

3. Goulielmakis, E. et al. Attosecond control and measurement: lightwave electronics. Science 317, 769-775 (2007).

4. Baltuska, A. et al. Attosecond control of electronics processes by intense lightwaves. Nature 421, 611-615 (2003).

5. Kubler, C., Huber, R., Tubel, S. \& Leitenstorfer, A. Ultrabroadband detection of multi-terahertz field transients with GaSe electro-optic sensors: approaching the near infrared. Appl. Phys. Lett. 85, 3360-3362 (2004).

6. Auston, D. H. \& Cheung, K. P. Coherent time-domain far infrared spectroscopy. J. Opt. Soc. Am. B 2, 606-612 (1985).

7. Kaindl, R. A., Carnahan, M. A., Hagele, D., Lovenich, R. \& Chemla, D. S. Ultrafast terahertz probes of transient conducting and insulating phases in an electron-hole gas. Nature 423, 734-738 (2003).

8. Günter, G. et al. Sub-cycle switch-on of ultrastrong light-matter interaction. Nature 458, 178-181 (2009).

9. Chan, W. L., Deibel, J. \& Mittleman, D. M. Imaging with terahertz radiation. Rep. Prog. Phys. 70, 1325 (2007).

10. Rungsawang, R., Ueno, Y., Tomita, I. \& Ajito, K. Angle-dependent terahertz time-domain spectroscopy of amino acid single crystals. J. Phys. Chem. B 110, 21259-21263 (2006).

11. Jördens, C. et al. Terahertz spectroscopy to study the orientation of glass fibres in reinforced plastics. Compos. Sci. Technol. 70, 472-477 (2010).
12. Fischer, B., Hoffman, M., Helm, M., Modjesch, G. \& Jepsen, P. U. Chemical recognition in terahertz time-domain spectroscopy and imaging. Semicond. Sci Technol. 20, 246-253 (2005)

13. Wu, Q. \& Zhang, X. -C. Free-space electro optic sampling of terahertz beams Appl. Phys. Lett. 67, 3523-3525 (1995).

14. Faist, J. et al. Quantum cascade laser. Science 264, 553-556 (1994).

15. Köhler, R. et al. Terahertz semiconductor heterostructure laser. Nature 417, 156-159 (2002)

16. Barbieri, S. et al. Imaging with a THz quantum cascade laser using a Schottky diode mixer. Opt. Express 13, 6497-6503 (2005).

17. Rabanus, D. et al. Phase locking of a 1.5 Terahertz quantum cascade laser and use as a local oscillator in a heterodyne HEB receiver. Opt. Express 17, 1159-1168 (2009).

18. Williams, B. S., Kumar, S., Hu, Q. \& Reno, J. L. High-power terahertz quantumcascade lasers. Electron Lett. 42, 89-91 (2006).

19. Baltuska, A. et al. Phase-controlled amplification of few-cycle light pulses. IEEE J. Sel. Top. Quantum Electron 9, 972-989 (2003).

20. Jukam, N. et al. Terahertz amplifier based on gain switching in a quantum cascade laser. Nat. Photonics 3, 715-719 (2009).

21. Dreyhaupt, A., Winnerl, S., Dekorsy, T. \& Helm, M. High intensity terahertz radiation from a microstructured large-area photoconductor. Appl. Phys. Lett. 86, 121114 (2005).

22. Worrall, C. et al. Continuous wave operation of a superlattice quantum cascade laser emitting at 2 THz. Opt. Express 14, 171-181 (2006).

23. Kröll, J. et al. Phase-resolved measurements of stimulated emission in a laser. Nature 449, 698-701 (2007).

24. Jukam, N. et al. Investigation of spectral gain narrowing in bound-tocontinuum quantum cascades lasers using terahertz time domain spectroscopy. Appl. Phys. Lett. 93, 101115 (2008).

25. Jukam, N. et al. Terahertz time domain spectroscopy of phonon-depopulation based quantum cascade lasers. Appl. Phys. Lett. 94, 251108 (2009).

26. Parz, W. et al. Ultrafast probing of light-matter interaction in a midinfrared quantum cascade laser. Appl. Phys. Lett. 93, 091105 (2008).

27. Dhillon, S. S. et al. Integrated terahertz pulse generation and amplification in quantum cascade lasers. Appl. Phys. Lett. 96, 061107 (2010).

28. Khuen, W. et al. Ultrafast phase-resolved pump-probe measurements on a quantum cascade laser. Appl. Phys. Lett. 93, 151106 (2008).

29. Wang, C. Y. et al. Mode-locked pulse from mid-infrared quantum cascade lasers. Opt. Express 17, 12929-12943 (2009).

30. Barbieri, S. et al. $3 \mathrm{GHz}$ direct modulaton of terahertz quantum cascades. Appl. Phys. Lett. 91, 143510 (2007).

31. Gaal, P., Raschke, M. B., Reimann, K. \& Woerner, M. Measuring optical frequencies in the $0-40 \mathrm{THz}$ range with non-synchronized electro-optic sampling. Nat. Photonics 1, 577-580 (2007).

32. Barbieri, S. et al. Phase-locking of a $2.7 \mathrm{THz}$ quantum cascade laser to a modelocked ebrium-doped fiber laser. Nat. Photonics 4, 636-640 (2010).

\section{Acknowledgments}

This work was financially supported by DGA, CNano and ANR. The LPA-ENS is a

'Unité Mixte de Recherche Associée au CNRS UMR8551 et aux Universités Paris 6 et 7'.

\section{Author contributions}

D.O. and R.R. collected the experimental data. N.J conceived and set up the experiment Preliminary measurements were taken by N.J and D.O. J.M prepared samples. Sample growth was performed by X.M. Samples were processed by P.F. S.B and C.S. gave insight on the phase locking of QCLs. The paper was written and the data were interpreted by N.J, J.T, S.D., D.O., R.R., S.B. and C.S. All work was coordinated and overseen by J.T. and S.D.

\section{Additional information}

Supplementary Information accompanies this paper on http://www.nature.com/ naturecommunications

Competing financial interests: The authors declare no competing financial interests

Reprints and permission information is available online at http://npg.nature.com/ reprintsandpermissions/

How to cite this article: Oustinov, D. et al. Phase seeding of a terahertz quantum cascade laser. Nat. Commun. 1:69 doi: 10.1038/ncomms1068 (2010).

License: This work is licensed under a Creative Commons Attribution-NonCommercialNoDerivative Works 3.0 Unported License. To view a copy of this license, visit http:// creativecommons.org/licenses/by-nc-nd/3.0/ 